\title{
"Weil es eine der Geheimnisse von Rom": Die Mozarts als verhinderte Überbringer von Allegris Miserere
}

\section{„weil es eine der Geheimnisse von Rom": The Mozarts as Prevented Bearers of Allegris Miserere}

Thomas Hochradner / thomas.hochradner@moz.ac.at University of Music and Dramatic Arts Mozarteum Salzburg, AT

\begin{abstract}
Since the times of Renaissance, Italian culture and arts had broadly reached the regions north of the Alps. To have poets, painters, singers and chapel masters from Italy at court became one of the main features of absolutism. However, only when soon after 1700 the War of Spanish Succession raged and swapped lots of Italian instrumentalists to the German territories, the Italian orchestra style began to dominate, embracing all music with its very specific characters. Thus the Mozarts, when listening to Allegri's famous Miserere in Rome in 1770, must have felt surprised by its Falsobordone setting. Yet other devices of the performance, being baroque in their origin, should have been quite common to them. Against this background, a re-evaluation of the often commented 'wonder' of Wolfgang Amadés by heart-transcription is suggested.
\end{abstract}

\section{Keywords}

Mozart Father and Son, Gregorio Allegri's Miserere setting, Rome, Cappella Sistina, Tradition into $18^{\text {th }}$ century Austria, Salzburg experiences of Holy Week performances 
„Kayser Leopold [I., geb. 1640, Kaiser 1658-1705], welcher nicht nur ein Liebhaber und Gönner der Musik war, sondern auch selbst gut komponierte, befahl seinem Gesandten zu Rom, vom Pabste die Erlaubniß zu erbitten, daß er eine Abschrift von dem berühmten Miserere des Allegri zum Gebrauch der Kaiserlichen Kapelle zu Wien nehmen dürtte: als er diese Erlaubniß erhalten hatte; schrieb der päbstliche Kapellmeister es für ihn ab, und schickte es dem Kaiser zu, der damals einige von den größten Sängern seiner Zeit in Diensten hatte. Allein der Geschicklichkeit dieser Sänger ungeachtet, that diese Komposition der Erwartung des Kaisers und seines Hofes, als sie aufgeführt ward, so wenig Genüge, daß er den Schluß machte, der päbstliche Kapellmeister hätte ihn hintergangen, und, um seinen Schatz, als ein Geheimniß für sich zu behalten, eine Komposition unterschoben. Der Kaiser war darüber sehr aufgebracht, und schickte einen Kurier an Se. [Seine] Heiligkeit, sich über den Kapellmeister zu beschweren, der deswegen in Ungnade fiel, und sogleich abgesetzt ward. Der Pabst war durch den vorgeblichen Betrug seines Kapellmeisters so sehr beleidigt, daß er ihn lange Zeit hindurch weder sehen, noch seine Vertheidigung hören wollte; doch endlich übernahm es einer von den Kardinälen, Fürsprache für ihn zu thun, und sagte $\mathrm{Se}[\mathrm{r}]$. Heiligkeit, daß die in der päbstlichen Kapelle übliche Art zu singen, vornehmlich bey diesem Miserere so beschaffen wäre, daß sie nicht in Noten ausgedrückt werden, oder anders als durchs Exempel könne gelehrt, und an andern Orten eingeführt werden; weswegen diese Komposition, wäre sie auch noch so richtig abgeschrieben, ihrer Wirkung verfehlen müßte, sobald man sie anderswo aufführte. Se. Heiligkeit verstund keine Musik, und konnte gar nicht begreifen, wie die nehmlichen Noten an verschiedenen Orten so verschieden klingen könnten; indessen befahl er doch seinem Kapellmeister, eine schriftliche Vertheidigung einzugeben, welche nach Wien gesandt werden sollte: dies geschah [...]“1

1 BURNEY, Charles. Tagebuch einer Musikalischen Reise durch Frankreich und Italien. Hamburg: Bode, 1772. Faksimile-Nachdruck, Hrsg. Christoph Hust. Kassel u.a.: Bärenreiter, 2003 (Documenta Musicologica, Erste Reihe: Druckschriften-Faksimiles 19), S. 209-211. - Während sich im heute in der Musiksammlung der Österreichischen Nationalbibliothek verwahrten Bestand der Wiener Hofmusikkapelle keine ältere Handschrift des Allegri'schen Miserere erhalten hat, existiert - worauf mich Janice Stockigt freundlicherweise hinwies - eine leicht modifizierte Teilabschrift aus den 1730er Jahren (wenn auch demnach nicht der gegenständlichen, aus Rom nach Wien gesandten Kopie) von der Hand Jan Dismas Zelenkas, die in der Musikabteilung der Sächsischen Landesbibliothek in Dresden verwahrt wird (Signatur Mus. 2-E-12); vgl. dazu HORN, Wolfgang. Die Dresdner Hofkirchenmusik 1720-1745: Studien zu ihren Voraussetzungen und ihrem Repertoire. Kassel u.a.: Bärenreiter - Stuttgart: Metzler, 1987, S. 106ff. - Zwei handschriftliche Überlieferungen des Allegri'schen Miserere im Bestand der Musiksammlung der Österreichischen Nationalbibliothek, die aus dem späten 18. (Mus. Hs. 19.452) bzw. 19. Jahrhundert stammen und deren letztere (Mus. Hs. 15.604, verm. nach 1836) auch eine mit Verzierungen aktualisierte Fassung (so gesungen seit 1824) sowie eine weitere in der Singpraxis des Salzburger Doms (wo das Stück offenbar mit Orgelbegleitung ausgeführt wurde) bietet, bespricht anhand von Abschriften aus dem 19. Jahrhundert in der Bischöflichen Zentralbibliothek Regensburg, Proskesche Musikabteilung (PR-M Allegri 26 sowie C 104) Schiltz, Katelijne. Gregorio Allegris Miserere in Reiseberichten des 18. und 19. Jahrhunderts. In Prinzenrollen 1715/16. Wittelsbacher in Rom und Regensburg. Hrsg. Andrea und Jörg Zedler. München, 2016, S. 223-256, Anmerkungen S. 372-377: 231ff. bzw. 374. - In der Musiksammlung der Österreichischen Nationalbibliothek (Wien), die das historische Archiv der Wiener Hofmusikkapelle enthält, wird neben Mus. Hs. 19.542 (der Vorlage zu Pr-M Allegri 26, die wiederum nach dem Druck Milano: Giovanni Battista Giussani s. a., einer Druckerei, die bereits vor 1800 tätig war, angefertigt wurde) unter Mus. Hs. 19.451 eine weitere, vollständige Partiturabschrift des Allegri'schen Miserere samt Stimmensatz für die beide ersten Sätze und einem Ablauf für den Vortrag des Stückes aufbewahrt, ebenfalls aus dem späten 18. Jahrhundert datierend. Eine dritte, ebenfalls vollständige Partiturabschrift aus derselben Zeit findet sich unter Mus. Hs. 15.849. - Im Archiv der Erzdiözese Salzburg, Dommusikarchiv, hat sich eine Abschrift des Werkes erhalten, eine Partitur (M. N. 115,1an) aus der Sammlung und in der Handschrift von Johann Mederitsch (1752-1835, Kapellmeister und Freund von Mozarts jüngerem Sohn Franz Xaver alias Wolfgang Amadeus, dem er seinen Nachlass vermachte), die ein von Aloys Fuchs (1799-1853) verfasstes Etikett trägt. Möglicherweise ist nicht Leopold I., sondern Joseph II. gemeint. 
Charles Burney überliefert diese Anekdote in seinem Tagebuch einer Musikalischen Reise; man darf annehmen, dass sie weit verbreitet war, dass sie auch der belesene Leopold Mozart bereits kannte, als er mit seinem Sohn 1769 zur ersten italienischen Reise aufbrach. Am Mittwoch der Karwoche 1770 ergab sich dann Gelegenheit zu einer spektakulären musikalischen Leistungsschau: der 14-jährige Wolfgang Amadé Mozart hört das legendäre Miserere von Gregorio Allegri (1582-1652) und schreibt es danach aus dem Gedächtnis auf. Beginnend mit der frühen Biographik wurde dieses sporadische Ereignis immer wieder als Wunder und als beredtes Zeugnis von Mozarts Genialität beschrieben. Erstaunlich nüchtern liest sich dagegen, was seine Schwester, Maria Anna „Nannerl“ Mozart, aus ihrer Erinnerung über das damalige Geschehen berichtet:

\begin{abstract}
„Mittwoch nachmittag verfiegten sie [Vater und Sohn Mozart] sich also gleich in die Capellam Sixtinam, um das so berufene Miserere zu hören. und da der Sage nach, solches abcopieren zu lassen unter der excommunication der Päbst: Musick sollte verbotten seyn, so nahm sich der Sohn vor, solches wohl zu hören, und dann aufzuschreiben. daß geschahe auch, wie er nach Hause kam, schrieb er es auf, den folgenden tag gieng er wieder hin, hielt seinen aufsatz in hut, um zu bemerken, ob er es getrofen oder nicht. Allein es wurde ein anderes Miserere gesungen. Am Charfreytag wurde das nehmliche gemacht, nachdem er nach Hause kam, machte er da und dort eine Ausbesserung, dann war es fertig. Daß wurde nun bald in Rom bekannt, Er muste es in einer academie beym Clavier singen. der Castrat christofori, der es in der Capella sang war zugegen."
\end{abstract}

Eine erste Korrektur ist notwendig: Die Mozarts eilten am Nachmittag des 11. April 1770 keineswegs schnurstracks in die Sixtinische Kapelle, sondern besuchten davor Albert Alexander von Mölk, einen Sohn des Salzburger Hofkanzlers, im Collegium Germanicum nahe der Piazza Navona. ${ }^{3}$ Danach erst wandten sie sich dem Vatikan zu, um gleich vielen Rom-Touristen des 18. und 19. Jahrhunderts dem berühmten Allegri'schen Miserere zu lauschen. ${ }^{4}$ Friedrich von Schlichtegroll, der Empfänger von Nannerls Zeilen, der sich für seinen Mozart-Nekrolog ausführlich bei der Schwester erkundigt hatte, übernahm das „also gleich“ und war dann der Erste, der das Ereignis hochspielte, indem er Nannerls Erinnerungen hinzufügte, dass Christofori „durch sein Erstaunen Mozarts

2 Mozart. Briefe und Aufzeichnungen. Gesamtausgabe in 7 Bänden. Hrsg. Internationalen Stiftung Mozarteum Salzburg, gesammelt von Wilhelm Adolf Bauer und Otto Erich Deutsch, auf Grund deren Vorarbeiten erläutert von Joseph Heinz Eibl. Kassel u.a.: Bärenreiter, 1962-1975 [im Folgenden Bauer-Deutsch], Bd. IV, Nr. 1212, Z. 260-271.

3 ANGERMÜLLER, Rudolph, unter Mitarbeit von GEFFRAY, Geneviève. Delitiae Italiae. Mozarts Reisen in Italien. Bad Honnef: Bock, 1994, S. 90.

4 AMANN, Julius. Allegris Miserere und die Aufführungspraxis in der Sixtina nach Reiseberichten und Musikhandschriften. Regensburg: Pustet, 1935 (Freiburger Studien zur Musikwissenschaft [Fribourg, CH] 4), S. 31. - Für eine neuere Studie s. Bryam-Wigfield, Ben. Miserere mei, Deus. Gregorio Allegri. A Quest for the Holy Grail? (1996). Elektronisch verfügbar unter http://www.ancientgroove.co.uk/essays/AllegriBook.pdf (aufgerufen am 3. November 2017). - Das ideelle Moment der Reiseberichte des 19. Jahrhunderts diskutiert, mit einem Blick auf „Parameter der romantischen Musikästhetik“, Heidrich, Jürgen. „... Wie auf Schwanenflügeln getragen“. Zur Bedeutung der römischen Miserere-Rezeption für die deutsche literarische Romantik, Analecta Musicologica 33, 2004, S. 475-486, Zitat S. 481. 
Triumph vollkommen machte“. ${ }^{5}$ Bis zum heutigen Tag legte die Begebenheit an legendären Zügen weiter zu; in einer immerhin - aber zu Unrecht - als seriös beworbenen Mozart-Biographie aus dem Jahr 2006 heißt es:

„Das [die Aufführung des Allegri'schen Miserere durch die päpstliche Kapelle] war für Mozart eine herrliche Gelegenheit, um sein herausragendes musikalisches Gedächtnis zu zeigen, dachte Vater Mozart. Rom würde eine Sensation haben und der Name Mozart mit einem Schlag berühmt sein! Sein Plan war: Wolfgang solle gut aufpassen, sich die Musik merken und dann rasch, kaum dass er aus der Kapelle draußen war, alles nach dem Gedächtnis aufschreiben. Schließlich war eine Niederschrift der Noten aus dem Gedächtnis ja nicht verboten. So etwas hielt bei dieser komplizierten Partitur ohnehin niemand für möglich. [...] Vater Mozart tat alles, um diese Sensation in Rom bekannt zu machen. [...]“6

Allerdings, so vollends die Maße menschlicher Vorstellungskraft übersteigend war Mozarts musikalische Gedächtnisleistung nicht. Die familiäre Lesart bezeugt es. Nahezu marginal berichtet denn auch Leopold Mozart seiner Frau Anna Maria am 14. April 1770 aus Rom nach Salzburg:

\begin{abstract}
„Du wirst vielleicht oft von dem berühmten Miserere in Rom gehört haben, welches so hoch geachtet ist, daß den Musicis der Capellen unter der excommunication verbotten ist eine stimme davon. aus der Capelle weg zu tragen, zu Copieren, oder iemanden zu geben. Allein, wir haben es schon. der Wolfg: hat es schon aufgeschrieben, und wir würden es in diesen Briefe nach Salzb: geschickt haben, wenn unsere Gegenwarth, es zu machen, nicht nothwendig wäre; allein die Art der production muß mehr dabey thun, als die Composition selbst, folglich werden wir es mit uns nach hause bringen, und weil es eine der Geheimnisse von Rom ist, so wollen wir es nicht in andere Hände lassen, ut non incurremus mediate vel immediate in Censuram Ecclesiae."
\end{abstract}

Tatsächlich handelt es sich bei Allegris doppelchörigem Miserere aus der ersten Hälfte des 17. Jahrhunderts ${ }^{8}$ - eine von vielen Vertonungen des 50. Psalms Miserere mei Deus ${ }^{9}$ - um einen zwar simplen, doch wirkungsvoll schlichten Satz, den das musikalisch geschulte Gehör eines Hochbegabten durchaus behalten kann. Die Komposition folgt dem

5 Vgl. Vetter, Isolde. Mozarts Nachschrift des Allegrischen „Miserere“: Ein Gedächtnis-,wunder‘. In Musik als Text. Bericht über den Internationalen Kongreß der Gesellschaft für Musikforschung Freiburg im Breisgau 1993. Hrsg. Danuser, Hermann -Plebuch, Tobias. Bd. 2: ,Freie Referate‘, Kassel u.a.: Bärenreiter 1998, S. 144-147: 147.

6 Hamann, Brigitte. Mozart. Sein Leben und seine Zeit. Wien: Ueberreuter 2006, S. 75f.

7 Bauer-Deutsch (wie Anm. 2), Bd. I, Nr. 176, Z. 46-55 (Hervorhebung im Original).

8 Allegris Miserere muss zwischen 1630 und 1652 entstanden sein, als der Komponist als Sänger in der Cappella Sistina tätig war. Vermutet wird das Jahr 1638 - siehe LÜTTEKEN, Laurenz. Perpetuierung des Einzigartigen: Gregorio Allegris ,Miserere‘ und das Ritual der päpstlichen Kapelle. In Barocke Inszenierung. Hrsg. Imorde, Joseph -Neumeyer, Fritz - Weddigen, Tristan. Emsdetten - Zürich: Edition Imorde, 1999, S. 136-145: 139.

$9 \quad$ Vertonungen des Bußpsalms Miserere wurden dazumal im Offizium der letzten drei Kartage gesungen, in der Sixtinischen Kapelle zum Abschluss der Tenebrae-Liturgie, die am Gründonnerstag, Karfreitag und Karsamstag Matutin und Laudes umfasste. - Art. Miserere mei Deus. In Riemann Sachlexikon Musik. Hrsg. Gurlitt, Wilibald - Eggebrecht, Hans Heinrich. Mainz u.a.: Schott ${ }^{12} 1967$, Nachdruck Mainz: Schott 1996, S. 576. 
Alternatim-Prinzip; während die geraden Verse einstimmig, rezitiert auf einem Psalmton erklingen, erscheinen die ungeraden Verse in einer von rezitierenden Abschnitten durchsetzten, akkordisch dominierten mehrstimmigen Passage: in einem so genannten ,Falsobordone'-Satz. Im Wechsel werden für die ungeraden Verse ein fünfstimmiger (für die Verse 1, 5, 9, 13, 17) und ein vierstimmiger Satz (für die Verse 3, 7, 11, 15 und 19) in g-Dorisch vorgetragen. Diese Aufteilung war in Rom und vielerorts sonst bei figuralen Miserere die Regel. Ungewöhnlich und vor Allegris Vertonung nicht nachzuweisen ist hingegen das effektivere Ende: Der letzte, zwanzigste Vers beginnt mit dem fünfstimmigen Satz und endet in einem neunstimmigen Schlussabschnitt. ${ }^{10}$

Mozart hörte also den ersten Satz sechsmal, den zweiten fünfmal, den Schlussabschnitt einmal. In Frage steht, ob er alle mehrstimmigen Teile des Allegri'schen Miserere aufzeichnete, also auch den neunstimmigen Schluss. Unergründlich bleibt außerdem, inwieweit Mozart einen Rahmensatz skizzierte oder darüber hinaus auf die Aufführungspraxis der Cappella Sistina einging, ${ }^{11}$ die Allegris Komposition mit etlichen Verzierungen und Diminutionen interpretierte. ${ }^{12}$ Bedenkt man dazu, dass Mozart zwei Tage nach dem ersten Hören noch Korrekturen anbrachte, verblasst die Aura des Unbegreiflichen. Das Wunder nähert sich dem fortgeschrittenen Gehördiktat - ungeachtet der Tatsache, dass Mozart sein phänomenales Hörgedächtnis späterhin mehrfach in diversen Aufgriffen unter Beweis gestellt hat.

Dass Leopold Mozart die gesamte Aktion geplant hatte, ist nicht von der Hand zu weisen. Nicht nur für das Vorantreiben der Popularität Wolfgangs vor Ort, in Rom, sondern durchaus auch ein effektives Echo der Reiseerlebnisse in Salzburg sollte er dabei im Auge gehabt haben. Denn Leopold wusste nur zu gut, dass sich über das Medium Brief die lokale Öffentlichkeit erreichen und staunen machen ließ und sollte demnach kalkuliert haben, dass der eben zitierte Brief nicht nur an Frau und Tochter gelangen, sondern auch im Freundeskreis der Mozarts gelesen und sein Inhalt weiter kolportiert werden würde. Diesmal war das Aufsehen allerdings des Guten zu viel - ein Zeitungsartikel, der nicht erhalten ist, schilderte die Mozarts in Gefahr, die Ungnade des Papstes und die Exkommunikation heraufzubeschwören. Eine Gefahr für die Anstellung des Vaters am fürsterzbischöflichen Hof, generell für das Ansehen der Familie, lag in der Luft. Nun beeilte sich Leopold, den ehrsamen Ruf der Reisenden in Salzburg zu behaupten:

„Da wir den Articul wegen dem Miserere gelesen, haben wir alle beyde hell lachen müssen. Es ist desswegen gar nicht die mündeste sorge. Man macht ander Orts mehr daraus. ganz Rom weis es; und selbst

10 AMANN (wie Anm. 4), S. 1f.; MARX-WEBER, Magda. Römische Vertonungen des Psalms Miserere im 18. und frühen 19. Jahrhundert. Hamburger Jahrbuch für Musikwissenschaft 8, 1985, S. 7-43: 11; dies., Die Tradition der Miserere-Vertonungen in der Cappella Pontificia. In Collectanea. II: Studien zur Geschichte der päpstlichen Kapelle. Hrsg. Janz, Bernhard. Città del Vaticano: Biblioteca Apostolica Vaticana 1994 (Capellae apostolicae sixtinaeque collectanea acta monumenta 4), S. 265-288: 266.

11 Vgl. dazu auch ENGELS, Stephan [Stefan]. W. A. Mozart ed il „Miserere“ di G. Allegri. In Il Teatro di Mozart a Roma. Ausstellungskatalog der Biblioteca Vallicelliana. Hrsg. Ministerio per i Beni Culturali e Ambientali. Rom: Ufficio Centrale per i Beni Librari e gli Ist. Culturali 1991, S. 229-232: 232.

12 Diese waren allerdings im Zuge einer erneuten Niederschrift des Allegri'schen Miserere, die 1731 erfolgte, wahrscheinlich kodifiziert worden. AMANN (wie Anm. 4), S. 2, 5, 26 . 
der Pabst [Klemens XIV., geb. 1705, Papst 1769-74] weis es, daß der Wolfg: das Miserere geschrieben. Es ist gar nichts zu beförchten: es hat ihm vielmehr grosse Ehre gemacht, wie du in kurzem hören wirst. ${ }^{13}$ $d u$ sollst absolute den Brief aller ort lesen lassen, und solches $S^{r}$ : Hf. Gden [Seiner Hochfürstlichen Gnaden, also Fürsterzbischof Schrattenbach] zu wissen machen. "14

Tatsächlich kann die Gefahr der Exkommunikation längst nicht so groß gewesen sein, wie es Leopold brieflich unvorsichtiger Weise angedeutet hatte. Schon damals muss eine zwar überschaubare, aber eben doch gewisse Zahl von Abschriften existiert haben. ${ }^{15}$ Noch im selben Jahr 1770 reiste der eingangs zitierte Charles Burney, emsig recherchierender Reiseliterat in Sachen Musik - versehen mit einem Empfehlungsschreiben von Padre Martini, der Bologneser Autorität - nach Rom, fand Kontakt zum Kapellmeister der päpstlichen Kapelle Giuseppe Santarelli und erhielt vom Ersten Bibliothekar des Vatikans, Kardinal Alessandro Albani, die uneingeschränkte Erlaubnis zur Benützung des Musikarchivs. Albani war „als Mäzen und Förderer eine zentrale Figur für viele Musikerkarrieren des 18. Jahrhunderts"16 - die Freigabe des Archivs für Burney ist mithin als Einwilligung der Kurie zur Verbreitung darin enthaltener Materialien zu verstehen. Wie zu erwarten, galt das besondere Interesse Burneys dem ,berühmten“ Miserere; er kam an die Noten heran, es ist sogar wahrscheinlich, dass Burney, obwohl im November, Allegris Miserere von der päpstlichen Kapelle vorgesungen wurde. Dass die Hermetik des Zugangs zu diesem Werk zur Zeit des Mozart'schen Aufenthalts in Rom bereits aufgebrochen war, bestätigt schließlich die von Burney nur ein Jahr später besorgte Drucklegung des Miserere von Allegri ${ }^{17}$ zusammen mit dem zweiten damals in der Cappella Sistina gebräuchlichen Miserere von Tommaso Baj (ca. 1650-1714) ${ }^{18}$ - jenem Werk, das die Mozarts am Gründonnerstag 1770 zu hören bekommen haben.

13 Leopold Mozart spielt hier auf die Verleihung des, Ordens vom Goldenen Sporn“ durch den Papst an.

14 Brief Leopold Mozarts vom 19. Mai 1770 aus Neapel an seine Frau Anna Maria nach Salzburg; BauerDeutsch (wie Anm. 2), Bd. I, Nr. 184, Z. 73-79 (Hervorhebung im Original); kommentiert Bd. V, S. 256.

15 BURNEY (wie Anm. 1), S. 208f. - Ab dem letzten Drittel des 18. Jahrhunderts entstanden dann zahlreiche handschriftliche Kopien; vgl. AMANN (wie Anm. 4), S. 96f.; KEIL, Klaus. „Chi si canta nella Cappella Sistina“. Quellen zur Rezeption des Repertoires der päpstlichen Kapelle. In Festschrift für Winfried Kirsch zum 65. Geburtstag. Hrsg. Ackermann, Peter - Kienzle, Ulrike - Nowak, Adolf. Tutzing: Schneider, 1996, S. 130-142: 137-142.

16 Brandenburg, Daniel. „Die Hoheit und Pracht dieses Schauspiels“. Zu Niccolò Jommellis Opera seria Demofoonte. In Niccolò Jommelli. Demofoonte. Programmheft. Salzburg: Salzburger Pfingstfestspiele 2009, S. 30-34: 31.

17 La MUSICA / Che si Canta Annualmente / nelle FUNZIONI della SETTIMANA SANTA / nella CAPPELLA PONTIFICIA / Composta dal / PALESTRINA, ALLEGRI, e BAI / Raccolta e Pubblicata / da / CARLO BURNEY Mus. D. / Londra, Price 10:6 / Stampata per ROBERTO BRENNER, nella Strand / 1771.; siehe dazu Keil (wie Anm. 15), S. 131. - Das Werk hat sich sodann rasch in Mitteleuropa verbreitet: Robert Hugo verdanke ich den Hinweis, dass Allegris Miserere schon um 1820 in der Kirche des Ordens der Kreuzherren mit dem Roten Stern in Prag, im Übrigen in einer vielkehligen A-cappella-Besetzung, gesungen worden ist.

18 Eine jüngere, bald nach 1700 entstandene Vertonung des 50. Psalms, die in einer mit 1713 datierten Handschrift überliefert ist. Siehe Marx-Weber, Magda. Die Entwicklung des Karwochenrepertoires der päpstlichen Kapelle, insbesondere der Lamentationen. In dies. Liturgie und Andacht. Studien zur geistlichen Musik. Paderborn: Ferdinand Schöningh, 1999 (Beiträge zur Geschichte der Kirchenmusik 7), S. 216-233: 229. 


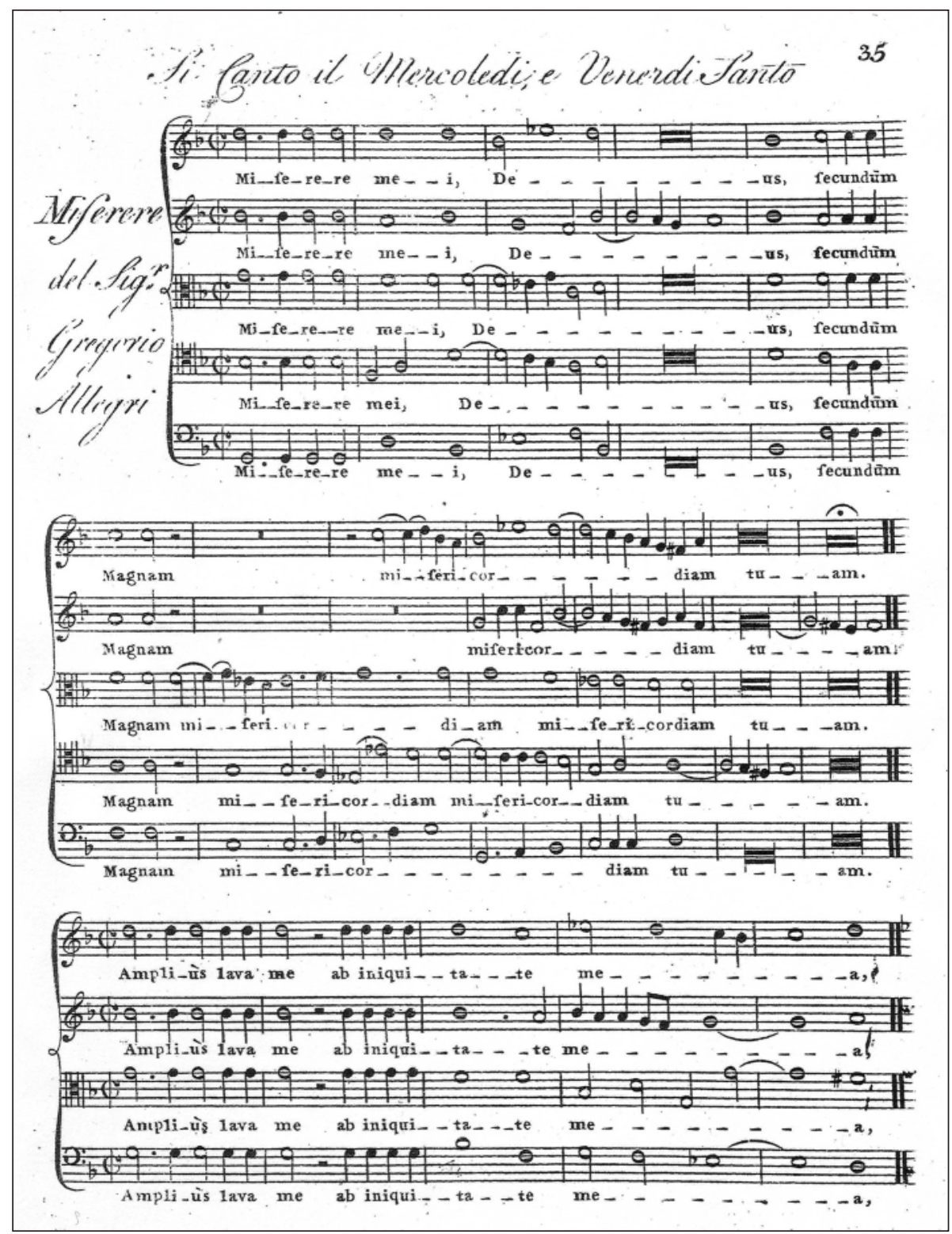

Abb. 1 Beginn des Miserere von Gregorio Allegri in der von Charles Burney 1771 besorgten Erstausgabe (La MUSICA / Che si Canta Annualmente / nelle FUNZIONI della SETTIMANA SANTA / nella CAPPELLA PONTIFICIA / Composta dal / PALESTRINA, ALLEGRI, e BAI / Raccolta e Pubblicata / da / CARLO BURNEY Mus. D. / Londra, Price 10:6 / Stampata per ROBERTO BRENNER, nella Strand / 1771, S. 35)

Überdies teilte Santarelli Burney Details zur Aufführungspraxis mit, die Leopold Mozarts Andeutungen entschlüsseln: 
„Dieß Stück, welches über hundert und funfzig Jahr, jährlich in der Charwoche in der päbstlichen Kapelle am Mittwochen und Charfreytage aufgeführet werden, und dem Ansehn nach so simpel ist, daß diejenigen, welche es bloß auf dem Papiere gesehen haben, sich wundern, woher seine Schönheit und Wirkung entstehen könne, hat seinen Ruhm mehr der Art, wie es aufgeführet wird, als der Komposition zu danken. Die nämliche Musik wird verschiedenemal mit verändertem Texte wiederholt, und die Sänger haben eine gewisse von Alters her überlieferte Art zu singen, gewisse Ausdrücke, gewisse hergebrachte Auszierungen [...] welche große Wirkung thun: Z.E. [zum Exempel] eine gemeinschaftliche Verstärkung oder Schwächung des Tons; die Beschleunigung oder Verzögerung des Takts bey gewissen Worten, und daß sie einige ganze Strophen geschwinder singen, als andere. "19

Danach lenkt Burney die Aufmerksamkeit in eine noch andere Richtung:

\begin{abstract}
„Doch müssen wohl einige von den großen Wirkungen, welche dieses Stück hervorbringt, eigentlich der Zeit, dem Orte und den feyerlichen Ceremonien zugeschrieben werden, welche bey der Aufführung gewöhnlich sind. Der Pabst und das ganze Conclave liegen kniend an der Erde, die Lichter der Kapelle und die Fackeln auf dem Geländer werden eins nach dem andern ausgelöscht; und der letzte Vers dieses Psalms wird von zwey Chören beschlossen, indem der Kapellmeister den Takt immer langsamer schlägt, und die Sänger die Harmonie bis zum völligen Schlusse ganz allmählig endigen oder vielmehr ausgehen lassen.
\end{abstract}

Es wird ebenfalls von ausgesuchten Sängern aufgeführt, welche viele Proben machen, vornehmlich des Montags in der Charwoche, welchen man dazu anwendet, das Stück oftmals zu probiren, und die Feinheiten der Ausführung zu lehren. "20

Freilich gilt es zu bedenken, dass Burney Anglikaner und womöglich mit den Gepflogenheiten der damaligen katholischen Liturgie - zumal einer selten ausgeübten - nicht allzu vertraut war. So waren die Fenster der Sixtinischen Kapelle verhängt ${ }^{21}$ und wurde nach dem Einzug des violett gekleideten Papstes, der keine Mitra trug, mit dem Ende der Matutin die erste Kerze gelöscht. Es folgten die Lamentationen des Jeremias und die Laudes und dann, nach der Antiphon Justificeris Domine, choraliter der 50. Psalm, also das Miserere. Während des anschließenden Canticum Zachariae Benedictus Dominus erhob sich der Papst vom Faldistorium und nun wurden nach und nach alle Kerzen verlöscht, die letzte nahm der Zeremoniar vom Leuchter und trug sie hinter den Altar. Nun erklang die Antiphon Christus factus est, der Papst und mit ihm alle Anwesenden, die Sänger ausgenommen, knieten nieder und jetzt erst kam das Miserere in Allegris Vertonung zur Ausführung. Am Schluss intonierte der Papst die Oratio Respice quaesumus Domine, deren letzte Sätze jedoch still gebetet wurden. Dann klopfte der Zeremoniar mit den Händen auf eine Altarstufe oder ein Holzbänkchen und die gesamte Gemeinde

19 BURNEY (wie Anm. 1), S. 206 f.

20 Ebenda, S. 207f.

21 HEIDRICH (wie Anm. 4), S. 479. 
hatte laut zu lärmen, ehe der Zeremoniar wieder eine Kerze anzündete, ein Zeichen sich von den Knien zu erheben und in Stille die Kapelle zu verlassen. ${ }^{22}$

Demnach wurde der Psalm Miserere bereits zu Beginn der Laudes choraliter abgesungen, was die Wirkung seiner figuralen Darbietung am Ende der Liturgie erhöht haben dürfte. Im Weiteren beeinflusste die herrschende Dunkelheit die Aufführung; die Sänger hatten auswendig zu singen - der Grund, warum die Verzierungen, die Temponahmen, die Textunterlegung von Jahr zu Jahr zuvor gut zu proben waren. ${ }^{23}$ Dies war das eigentliche Novum für die Mozarts, ein in seiner einmaligen Verbindung von Ort, Liturgie und Ausführung intransportables Moment. Folgerichtig kommentiert es Leopold als „eines der Geheimnisse von Rom“, konsequenter Weise hüteten die Mozarts Wolfgangs Transkription keineswegs als besonderen Schatz in ihrem Notenstapel. Dass sein Autograph des Allegri'schen Miserere verschwand - was seine Hörleistung für die Nachwelt wohl noch aufwertete - geschah vielleicht sogar, weil die Aufzeichnung doch nicht so gelungen war und deshalb irgendwann als unerheblich ausgeschieden wurde. ${ }^{24}$

Im Übrigen dürfte die Mozarts die rituelle Inszenierung des Geschehens in der Sixtinischen Kapelle wenig beeindruckt haben; ${ }^{25}$ denn der Ablauf der Tenebrae, die an den drei letzten Tagen der Karwoche stattfanden und bei denen Matutin und Laudes zusammengelegt waren, gestaltete sich überall nach einem eingeführten Formular. ${ }^{26}$ Seit dem

22 LÜTTEKEN (wie Anm. 8), S. 142, der als Quelle das Zeremonienbuch in der Edition von Marc Dykmans SJ benützt hat: DYKMANS, Marc. L'Euvre de Patrizi Piccolomini ou le cérémoniel papal de la première Renaissance. 2 Bände. Città del Vaticano: Biblioteca Apostolica Vaticana, 1980 (Studi e testi 293). - Darüber hinaus stützt sich Lütteken auf Marx-Weber, Römische Vertonungen (wie Anm. 10), S. $7 \mathrm{f}$.

23 Veränderungen schlossen sich dennoch nicht aus. Aus dem Jahr 1731 stammt eine Handschrift der Cappella Sistina, worin der fünfstimmige erste Chor Allegris deutlich verändert erscheint; vielleicht schon knapp zuvor war von einem Sänger der päpstlichen Kapelle, Giovanni Bordi, eine Handschrift angelegt worden, worin der fünfstimmige Chorsatz auf eben die selbe Weise verändert erscheint wie in der oben erwähnten, mit 1731 datierten Handschrift; eine weitere aus dem Jahr 1748 stammende Handschrift zeigt denselben Notentext; siehe dazu MARX-WEBER, Römische Vertonungen (wie Anm. 10), S. 11; dies., Die Tradition der MiserereVertonungen (wie Anm. 10), S. 273f. - Aus dem Jahr 1831 datiert jenes Verzierungsnotat, das Felix Mendelssohn Bartholdy während eines Rom-Aufenthaltes anfertigte; siehe dazu Amann (wie Anm. 4), S. $70 f$.

24 Was - nebenbei bemerkt - nicht entschuldigt, dass diese Mozart'sche ,Arbeit‘ selbst in der letzten Auflage des Köchel-Verzeichnisses unberücksichtigt geblieben ist.

25 Ganz anders also, als es den wenig später einsetzenden Reiseberichten, etwa von Friederike Brun (Tagebuch über Rom. Zürich: Orell, Füssli und Co. 1796), Elisa van der Recke (Tagebuch einer Reise durch einen Theil Deutschlands und durch Italien in den Jahren 1804 bis 1806. Bd. 2, Berlin: Nicolaische Buchhandlung 1815) und Wilhelm Heinse (Brief an Friedrich Heinrich Jacobi vom 13. März 1782, s. Sämtliche Werke. Bd. X. Leipzig: Insel, S. 55 - vgl. Jestremski, Margit. Heinses Werkstattnotizen zur Musik. In Wilhelm Heinse. Der andere Klassizismus. Hrsg. Bernauer, Markus - Miller, Norbert. Göttingen: Wallstein 2007, S. 268-278: 272; Hildegard von Hohenthal. Teil 1, Berlin: Voss 1795), zu entnehmen ist [für etliche Beispiele s. Amann (wie Anm. 4) und für eine Besprechung Schiltz (wie Anm. 1), S. 238-245]. All diese Autorinnen und Autoren empfanden die Liturgie offenkundig als ,ästhetisches Gesamtkonzept‘ und schilderten dementsprechend mehr Eindrücke als Umstände des Geschehens.

26 Grundsätzlich verlief die Tenebrae-Liturgie für gewöhnlich so: Drei Nokturnen zu je drei Lektionen (darunter die Lamentationen des Jeremias) folgten Versikel und Responsorium, ein still gebetetes Pater noster, drei Lesungen mit Responsorium (Matutin), fünf Psalmen, Versikel und Responsorium, das Canticum Zachariae Benedictus Dominus, die Antiphon Christus factus est, ein weiteres still gebetetes Pater noster, schließlich der Strepitus. 14 Lichter werden nach den einzelnen Psalmen verlöscht, die Altarkerzen während des Benedictus Dominus, die letzte Kerze wird unter den Altar gestellt - die Kirche liegt nun völlig im Dunkeln. Der Strepitus 
Mittelalter hatte sich das allmähliche Verlöschen der Kerzen über der Psalmenfolge eingebürgert, nach dem Tridentinum war die Liturgie auf den Vorabend verlegt worden. Wie der Theologiestudent Heinrich Pichler in seinem Tagebuch für den 1. April 1747 berichtet, geschah es dergestalt auch am Salzburger Dom: „Die Lamentationes singen die Vocalisten auf dem Chor oben, doch ohne Orgel oder einen anderen Instrument, sondern ganz allein die Versiculos singen auch die 2 Ministri sub-et diaconus. Die Lectiones singen die letzteren Chorherren. Das ,Benedictus ' wird partim choraliter partim mit der Capellnmusic gesungen und also auch das ,Miserere', aber solches in einem thraurigen Ton und sehr langsam. Auf die Letzt werden sogar die Liechter in denen Lampen oder Öllampern ausgelöschet. " 27 Auch wenn sonstige nähere Nachrichten fehlen, macht eine 1784 erlassene Ordinariatsvorschrift deutlich, dass all dies auch am Salzburger Dom so geschehen war. Nun aber heißt es, man solle darauf achten, „keine Kirche mehr, wie es an einigen Orten bisher üblich war, geflissentlich zu verfinstern", sei „dagegen aber, wann und wo es nothwendig wird, früh vor Anbruch des Tages und Abends bey eingehender Dämmerung, der innere Umfang des Gotteshauses besonders unter den Bögen, auf den Chören, in den Oratorien, Sakristayen u.a. zu Beseitigung aller sich etwa begebenden Unanständigkeiten hinlänglich zu beleuchten [...]“. ${ }^{28}$

Doch 1770 dominierten noch die Tentakel barocker Frömmigkeit, auch wenn sie gelegentlich - wie am Salzburger Dom - ein älteres a cappella-Repertoire für das Proprium missae miteinschlossen. Trotzdem muss die Mozarts, als sie am 11. April 1770 in der Sixtinischen Kapelle das Miserere von Allegri erwarteten, eine Distanz zur Gegenwart umfangen haben - entstammte doch deren Raumgestaltung der Renaissance ${ }^{29}$ und kam dazu noch eine unvertraute musikalische Ausführung auf sie zu. Denn gemessen an der längst währenden Intensität musikalischer Wanderschaft italienischer Provenienz auf der ,Nordroute ${ }^{630}$ bildet die Interpretation von Allegris Miserere durch die Cappella Sistina einen Ausnahmefall. Als mit und nach dem Spanischen Erbfolgekrieg zu Beginn des 18. Jahrhunderts zunehmend nicht nur Kapellmeister, Sängerinnen und Sänger, sondern auch Orchestermusiker den ,Markt‘ nördlich der Alpen entdeckt und geschwemmt

(Lärm) steht für das Erdbeben nach Christi Tod. Danach wird die Kerze unter dem Altar wieder hervorgeholt, als Symbol der nahen Auferstehung. Diese Liturgie wurde erst 1955 durch Papst Pius XII. modifiziert. Siehe dazu Tenebrae. Eintrag in http://en.wikipedia.org/wiki/Tenebrae, aufgerufen am 3. Juli 2009.

27 MARTIN, Franz. Vom Salzburger Fürstenhof um die Mitte des 18. Jahrhunderts. Teil 1. Mitteilungen der Gesellschaft für Salzburger Landeskunde 77/78, 1937/38, S. 1-48: 37.

28 Ordinariatsvorschrift welche in feyerlicher Begebung des 40. stündigen und zum Theil auch 7. stündigen Gebethes bey allen sowohl Säkular-als Regularkirchen des hohen Erzstiftes von nun an genau zu beobachten ist. A-Saes 1.2, 22/52. Zit. nach ZYBINA, Karina. Die Litaneien von Wolfgang Amadeus Mozart und die Salzburger Tradition. Diss. Universität Mozarteum Salzburg, 2017, S. 22f.

29 Zum Altar hin links werden Szenen aus dem Leben Moses' gezeigt, rechts Szenen aus dem Leben Christi; es handelt sich um Fresken, ausgeführt zwischen 1480 und 1483 von den prominentesten italienischen Malern dieser Zeit. Ursprünglich nahmen die Szenenfolgen ihren Ausgang von der Darstellung der Auffindung Moses' bzw. der Geburt Christi, die seitlich des Hochaltares angebracht waren. Beide Gemälde wurden - wie das eigentliche Altarbild, Die Himmelfahrt Mariens - zerstört, als Michelangelo Buonarotti im Auftrag von Papst Paul III. zwischen 1534 und 1541 die Altarwand mit Das Jüngste Gericht ausgestaltete.

30 Vgl. HOCHRADNER, Thomas. Musik im Zeitspiegel des Spanischen Erbfolgekriegs. In Festschrift Hellmut Federhofer zum 100. Geburtstag: Hrsg. Beer, Axel in Verbindung mit Gruber, Gernot - Schneider, Herbert. Tutzing: Schneider, 2011 (Mainzer Studien zur Musikwissenschaft 45), S. 131-144. 
hatten, war diese anlassspezifische Traditionsgebundenheit - zumal nicht modern ,daheim‘ und verborgen geblieben und überraschte Besucher wieder und wieder, wie bereits 1715 den reisenden Kavalier Johann Friedrich Armand von Uffenbach:

„Gegen abend fuhr nach dem St. Peter, alda in der capella paulina vor dem pabst und seiner clerisey das miserere von der capelle und allen castraten gesungen wurde, ich kam aber ein wenig zu spath so daß vor dem abscheulichen getränge nicht hinein kommen kunte außen aber hörte dem gesang ein wenig zu, die gravität des pabstes leidet nicht daß ein orgel oder instrument gespiehlet werde vor ihm in der Kirche, daher nur ein Chor der castraten, dießes miserere so erbärmlich und doch fürtrefflich sang, daß es einen recht zur andacht bewegte, es ist solches ein alt fränkisch coral music aber meisterlich und unvergleichlich gesezet, auch izo noch besser exequirt werden, der falschen und künstlichen thon waren ohnzahlig und das aushalten perfect einer resonanz der orgel gleich, so daß man geschwohren hätte es seyen keine menschen stimmen, sondern instrumente, alles war über das violet und schwarz behängt und in tiefer andacht. " ${ }^{11}$

Was für den homophonen Satz des Frühbarock gilt, trifft auch für den kontrapunktischen zu: Was nördlich der Alpen als „stile alla Palestrina“ gehandelt wurde, war über viele Stationen, nicht zuletzt Fux' Gradus ad Parnassum, zu einem Schulwerk verkrustet, das entweder gelehrig-brav befolgt oder verwässert, da kreativ weitergesponnen wurde. Peter Lüttig bringt diese Gemengelage auf den Punkt:

„Daß der Kaiserliche Hofkapellmeister [Fux] mit Palestrina die traditionelle Meinung seiner Zeit von Legende und synonymer stilistischer Beschreibung meint, dürfte klar sein: daß er es aus verständlicherweise traditionellen Erwägungen heraus tat [indem er in absolutistischer Gefolgschaft den Nestor, quasi ,Fürsten' der Kirchenmusik als Repräsentanten heranzog], wird schon dadurch einsichtig, daß von einer Gegenüberstellung des Palestrina-Stils zur zeitgenössischen Musizier- und Kompositionspraxis inhaltich in den Gradus keine Rede sein kann. "32

Aber die italienische polyphone kirchenmusikalische Tradition hatte in der Person von Padre Martini einen prominenten Fürsprecher gefunden, der ihre Merkmale - die imitatorische Durchwirkung eines Satzes, die Linearität als kompositorisches Prinzip nicht nur hochhielt, sondern auch allgemein belebte, zumal sie zu einem Kriterium für die Aufnahme in die Bologneser Accademia filarmonica avancierten. Bald nach dem Aufenthalt in Rom, vielleicht im Vorfeld der Aufnahmeprüfung, übte sich Mozart in Bologna mit einem eigenen, zwölftaktig konzipierten, achtsätzigen Miserere im strengen Satz. Wie aufgedeckt wurde, nicht ohne Probleme einer sinnfälligen Textunterlegung, doch - als ob die Singularität des Vorfalls in Rom zu unterstreichen wäre - mit Ausnahme der Alternatim-Anlage ohne jeden Rückgriff auf das Miserere Allegris. ${ }^{33}$ Und

31 PREUßNER, Eberhard. Die musikalischen Reisen des Herrn von Uffenbach. Aus einem Reisetagebuch des Johann Friedrich A. von Uffenbach aus Frankfurt a. M. 1712-1716. Kassel - Basel: Bärenreiter, 1949, S. 80.

32 LÜTTIG, Peter. Der Palestrina-Stil als Satzideal in der Musiktheorie zwischen 1750 und 1900. Tutzing: Schneider, 1994 (Frankfurter Beiträge zur Musikwissenschaft 23), S. 46.

33 Mehr scheint sich Mozart in diesem Miserere an eine jüngere römische Vertonungstradition des Psalms 
dennoch: beide Male reibt sich ein durchaus italophiles Verständnis von Kirchenmusik mit einem Traditionsgehalt, der nicht über die Alpen gedrungen war und den Leopold Mozart seinen Sohn nicht lehren konnte.

\section{Bibliography}

AMANN, Julius. Allegris Miserere und die Aufführungspraxis in der Sixtina nach Reiseberichten und Musikhandschriften. Regensburg: Pustet, 1935 (Freiburger Studien zur Musikwissenschaft [Fribourg, $\mathrm{CH}] 4$ ).

ANGERMÜLLER, Rudolph, collaborating GEFFRAY, Geneviève. Delitiae Italiae. Mozarts Reisen in Italien. Bad Honnef: Bock, 1994.

ANON., Miserere mei Deus. In Riemann Sachlexikon Musik. Ed. by Gurlitt, Wilibald - Eggebrecht, Hans Heinrich. Mainz et al.: Schott ${ }^{12} 1967$, Reprint Mainz: Schott, 1996, p. 576.

BRYAM-WIGFIELD, Ben. Miserere mei, Deus. Gregorio Allegri. A Quest for the Holy Grail? (1996). URL: http://www.ancientgroove.co.uk/essays/AllegriBook.pdf (accessed 3 November 2017).

BURNEY, Charles. Tagebuch einer Musikalischen Reise durch Frankreich und Italien. Hamburg: Bode 1772. Facsimile-Reprint, ed. by Hust, Christoph. Kassel et al.: Bärenreiter, 2003 (Documenta Musicologica, First Series: Druckschriften-Faksimiles 19).

ENGELS, Stephan [Stefan]. W. A. Mozart ed il „Miserere“ di G. Allegri. In Il Teatro di Mozart a Roma. Catalogue of an Exhibition in Biblioteca Vallicelliana. Ed. by Ministerio per i Beni Culturali e Ambientali. Rom: Ufficio Centrale per i Beni Librari e gli Ist. Culturali 1991, pp. 229-232.

HEIDRICH, Jürgen. „... Wie auf Schwanenflügeln getragen“. Zur Bedeutung der römischen Miserere-Rezeption für die deutsche literarische Romantik. Analecta Musicologica 33, 2004, pp. $475-486$.

HINTERMAIER, Ernst. Zur Urheberschaft des Introitus „Cibavit eos“ KV 44 (73u). Mozarts mißglückter Transkriptionsversuch einer mensural notierten Musik. Mozart-Jahrbuch 1991. Kassel et al.: Bärenreiter 1992, vol. I, pp. 509-517.

HOCHRADNER, Thomas. Musik im Zeitspiegel des Spanischen Erbfolgekriegs. In Festschrift Hellmut Federhofer zum 100. Geburtstag. Ed. by Beer, Axel collaborating Gruber, Gernot -Schneider, Herbert. Tutzing: Schneider 2011 (Mainzer Studien zur Musikwissenschaft 45), pp. 131-144.

HORN, Wolfgang. Die Dresdner Hofkirchenmusik 1720-1745. Studien zu ihren Voraussetzungen und ihrem Repertoire. Kassel et al.: Bärenreiter - Stuttgart: Metzler 1987, pp. 106-108.

JESTREMSKI, Margit. Heinses Werkstattnotizen zur Musik. In Wilhelm Heinse. Der andere Klassizismus. Ed. by Bernauer, Markus - Miller, Norbert. Göttingen: Wallstein 2007, pp. 268-278.

zu binden; vgl. MARX-WEBER, Magda. W. A. Mozart's Miserere K 85/73s. In All Kinds of Music: In Honour of Andrew D. McCredie. Hrsg. Strahle, Graham - Swale, David. Wilhelmshaven: Noetzel 1988, S. 117-120. Wie bei Mozarts Prüfungsarbeit zur Aufnahme in die Accademia filarmonica, der Antiphon Quaerite primum regnum Dei, gilt alle Aufmerksamkeit der Aneignung des kontrapunktischen Regelwerkes. Doch steht, wie Ernst Hintermaier gezeigt hat, im Hintergrund dieser kontrapunktischen Aktivität der Versuch einer musikgeschichtlichen Fundamentierung: Leopold Mozart hatte Sohn Wolfgang, wohl in der Absicht, die Aufnahme in die Bologneser Accademia zu erstreben, durch die Partitur-Abschrift eines Stimmensatzes zum Introitus Cibavit eos von Johann Stadlmayr im strengen Satz zu schulen gesucht - ein Unterfangen, das schließlich aus Unkenntnis mensuraler Notationspraxis abgebrochen wurde; vgl. HINTERMAIER, Ernst. Zur Urheberschaft des Introitus „Cibavit eos“ KV 44 (73u). Mozarts mißglückter Transkriptionsversuch einer mensural notierten Musik. In Mozart-Jahrbuch 1991. Kassel u.a.: Bärenreiter 1992, Teilband I, S. 509-517. 
KEIL, Klaus. „Chi si canta nella Cappella Sistina“. Quellen zur Rezeption des Repertoires der päpstlichen Kapelle. In Festschrift für Winfried Kirsch zum 65. Geburtstag. Ed. by Ackermann, Peter - Kienzle, Ulrike - Nowak, Adolf. Tutzing: Schneider 1996, pp. 130-142.

LÜTTEKEN, Laurenz. Perpetuierung des Einzigartigen: Gregorio Allegris ,Miserere‘ und das Ritual der päpstlichen Kapelle. In Barocke Inszenierung. Ed. by Imorde, Joseph -Neumeyer, Fritz - Weddigen, Tristan. Emsdetten - Zürich: Edition Imorde, 1999, pp. 136-145.

LÜTTIG, Peter. Der Palestrina-Stil als Satzideal in der Musiktheorie zwischen 1750 und 1900. Tutzing: Schneider, 1994 (Frankfurter Beiträge zur Musikwissenschaft 23).

FRANZ Martin. Vom Salzburger Fürstenhof um die Mitte des 18. Jahrhunderts. Teil 1. Mitteilungen der Gesellschaft für Salzburger Landeskunde 77/78, 1937/38, pp. 1-48.

MARX-WEBER, Magda. Die Entwicklung des Karwochenrepertoires der päpstlichen Kapelle, insbesondere der Lamentationen. In id., Liturgie und Andacht. Studien zur geistlichen Musik. Paderborn: Ferdinand Schöningh, 1999 (Beiträge zur Geschichte der Kirchenmusik 7).

MARX-WEBER, Magda. Die Tradition der Miserere-Vertonungen in der Cappella Pontificia. In Collectanea. II: Studien zur Geschichte der päpstlichen Kapelle. Ed. by Janz, Bernhard. Città del Vaticano: Biblioteca Apostolica Vaticana, 1994 (Capellae apostolicae sixtinaeque collectanea acta monumenta 4), pp. 265-288.

MARX-WEBER, Magda. Römische Vertonungen des Psalms Miserere im 18. und frühen 19. Jahrhundert. Hamburger Jahrbuch für Musikwissenschaft 8, 1985, pp. 7-43.

MARX-WEBER, Magda. W. A. Mozart's Miserere K 85/73s. In All Kinds of Music. In Honour of Andrew D. McCredie. Ed. by Strahle, Graham - Swale, David. Wilhelmshaven: Noetzel, 1988, pp. 117-120.

Mozart. Briefe und Aufzeichnungen. Complete Edition in 7 volumes. Ed. by Internationale Stiftung Mozarteum Salzburg, collected by Wilhelm Adolf Bauer and Otto Erich Deutsch, with comments by Joseph Heinz Eibl. Kassel et al.: Bärenreiter, 1962-1975.

PREUBNER, Eberhard. Die musikalischen Reisen des Herrn von Uffenbach. Aus einem Reisetagebuch des Johann Friedrich A. von Uffenbach aus Frankfurt a. M. 1712-1716. Kassel - Basel: Bärenreiter, 1949.

SCHILTZ, Katelijne. Gregorio Allegris Miserere in Reiseberichten des 18. und 19. Jahrhunderts. In Prinzenrollen 1715/16. Wittelsbacher in Rom und Regensburg. Ed. by Andrea and Jörg Zedler. München, 2016, pp. 223-256, enndnotes pp. 372-377.

VETTER, Isolde. Mozarts Nachschrift des Allegrischen „Miserere“: Ein Gedächtnis-,wunder‘. In Musik als Text. Bericht über den Internationalen Kongreß der Gesellschaft für Musikforschung Freiburg im Breisgau, 1993. Ed. by. Danuser, Hermann - Plebuch, Tobias. Vol. 2: ,Freie Referate', Kassel et al.: Bärenreiter, 1998, pp. 144-147.

ZYBINA, Karina. Die Litaneien von Wolfgang Amadeus Mozart und die Salzburger Tradition. Diss. University Mozarteum Salzburg, 2017. 
